\title{
The South African 24-hour movement guidelines for birth to 5 years
}

Physical activity, sedentary behaviour (which includes screen time) and sleep are collectively referred to as 'movement behaviours'. In young children, a healthy balance of these behaviours has been associated with better health and developmental outcomes, and in recent years, there has been a move towards integrated guidelines for these behaviours across the 24-hour day. This approach considers the natural integration of these behaviours in a 24-hour period, communicating a more cohesive message for parents, caregivers, teachers and practitioners. The 24-hour approach takes into consideration young children's health and development in the context of how they move throughout a usual day (a blend of sleeping, sitting, standing and different intensities of physical activity), and recognises that the whole day matters. ${ }^{[1]}$

Drawing on this evidence and the 24-hour approach, guidelines on young children's movement behaviours have been developed by a number of countries in recent years, including Canada, Australia and New Zealand. Global guidelines on these behaviours were launched by the World Health Organization in $2019,{ }^{[2]}$ also adopting the 24-hour approach. South Africa (SA) was the first low- to middleincome country to develop 24-hour movement guidelines for birth to 5 years, which were launched in December 2018. ${ }^{[1]}$

These guidelines provide evidence-based recommendations for young children's physical activity, sedentary (sitting) behaviour, screen time, and sleep. The process of developing these guidelines included stakeholder consultation ${ }^{[3]}$ to ensure that they were contextually relevant and appropriate for SA. The guidelines are available as a text document, and an infographic that is available in all 11 of SA's official languages (English infographic shown as Fig. 1). A song and video, ${ }^{[4]}$ entitled 'Woza, Mntwana', have also been produced as an additional creative resource to promote the guidelines.

The dissemination of the SA guidelines was carried out in 2019 with communitybased organisations (CBOs) working in early childhood development (ECD), using a 'trainthe-trainer' approach. Fifteen dissemination workshops were held in 7 of SA's 9 provinces, with a total of more than 320 attendees. Evaluation of this dissemination process, using qualitative and quantitative methods,

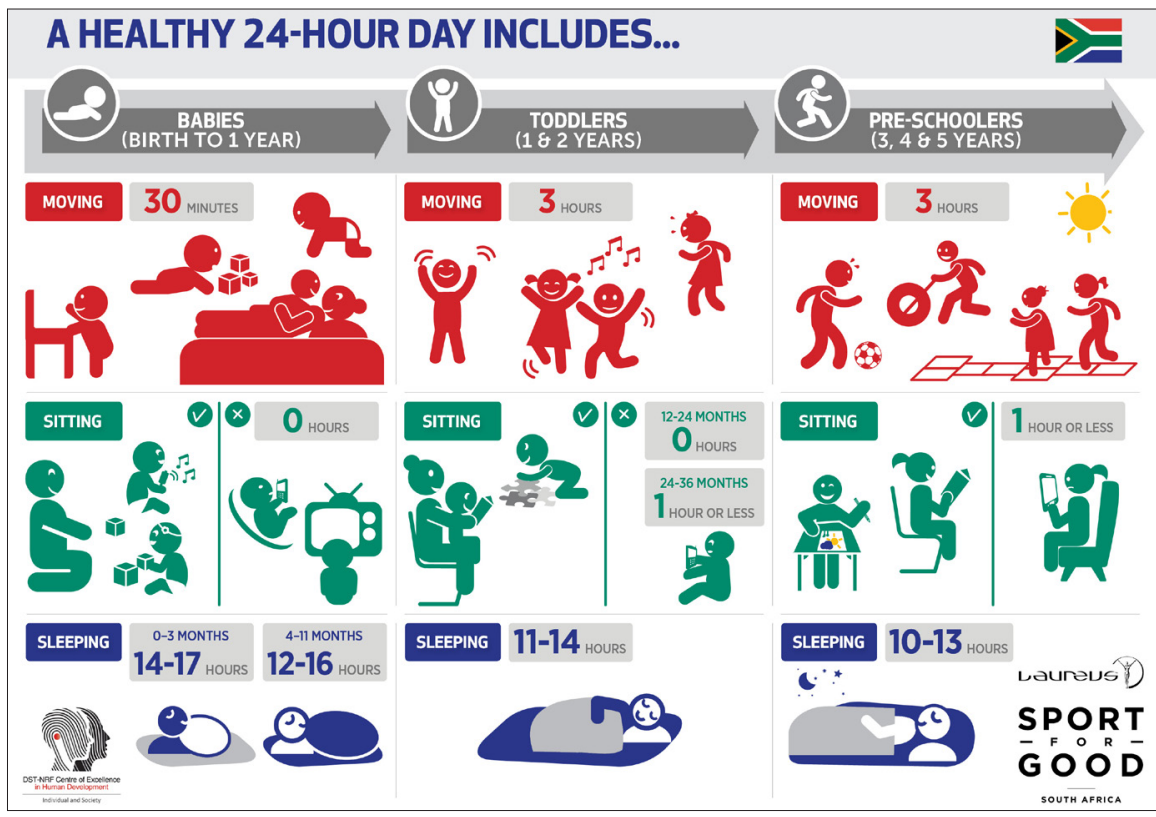

Fig. 1. South African 24-hour movement guidelines for birth to 5 years (English infographic). ${ }^{[1]}$

found that these workshops were a feasible and acceptable way to disseminate the guidelines at a community level. Follow-up of the dissemination found that $\mathrm{CBO}$ s were able to share these guidelines with the intended end-users of these guidelines, including parents, caregivers, teachers and practitioners, across a range of communities in SA. ${ }^{[5]}$

The original stakeholder consultation process highlighted a number of barriers to meeting the guidelines and competing priorities for those caring for young SA children, but also indicated overall positive perceptions of the guidelines. ${ }^{[3]}$ While the dissemination evaluation picked up on many of these same implementation challenges, ${ }^{[5]}$ the evaluation provided an additional opportunity to obtain insight into $\mathrm{CBO}$ and end-users' perceptions of the guidelines. Overall, $\mathrm{CBOs}$ and end-users had positive perceptions of the guidelines, and there was general agreement about the importance of these guidelines for the health and development of young children in SA. These findings support the adoption of a 24-hour approach to movement behaviour in the early years (and childhood more broadly), and that this approach contributes to a more cohesive message for parents, caregivers, teachers and practitioners. Perceptions of these guidelines can be an important indicator of the guidelines' acceptability and hence the extent to which these guidelines will be further disseminated by $\mathrm{CBO}$ and implemented by end-users. These insights are essential for a country such as SA where these types of guidelines are novel, and understanding perceptions of guidelines is valuable to guide the development and dissemination of similar guidelines addressing other health behaviours.

In terms of priorities for further intervention, research on the guidelines has highlighted that screen time and sleep are the two movement behaviours that should receive further attention in young SA children. This is supported by other evidence in the preschool age group, particularly in low-income, urban SA settings. ${ }^{[6,7]}$ While it can be challenging to change these behaviours in low-income, urban households, the research findings thus far have shown that caregivers of young children in these settings are often willing to try to make changes, especially if the focus is on child health and development - holistically, rather than just obesity or non-communicable disease prevention.

One of the key recommendations from this research on these guidelines is to promote their inclusion in other policy documents and initiatives in SA, and stakeholders have acknowledged the alignment of these guidelines with existing policy documents. Specifically, these guidelines are an excellent companion to the Road-to-Health booklet, since both emphasise the connection between 
caregivers and children, and the importance of love, play and talk to stimulate young children's development. The wide acceptability of the guidelines infographic and its availability in all 11 of SA's official languages make it an ideal accompaniment to resources already disseminated at scale to caregivers and stakeholders working with young children in SA. The infographic can be easily shared with child health practitioners, including paediatricians, general practitioners and allied health professionals, as well as community health workers, who in turn can share it with caregivers and other stakeholders.

In conclusion, the SA 24-hour movement guidelines for birth to 5 years should be disseminated and implemented for the promotion of early childhood health and development across all settings in SA. Establishing these healthy behaviours at a young age can help set children on their best trajectories for health and development, laying a good foundation for health, education and human capital outcomes later in life.

\section{Catherine E Draper}

SA MRC Developmental Pathways for Health Research Unit, School of Clinical Medicine, University of the Witwatersrand, Johannesburg, South Africa

catherine.draper@wits.ac.za
1. Draper CE, Tomaz SA, Biersteker L, et al. The South African 24-Hour Movement Guidelines for Birth to 5 Years: An integration of physical activity, sitting behavior, screen time, and sleep. J Phys Act Health 2020;17(1):109119. https://doi.org/10.1123/jpah.2019-0187

2. World Health Organization. Guidelines on physical activity, sedentary behaviour and sleep for children under 5 years of age. https://www.who.int/ publications/i/item/9789241550536 (accessed 17 June 2021).

3. Tomaz SA, Okely AD, van Heerden A, Vilakazi K, Samuels M-L, Draper CE. The South African 24-Hour Movement Guidelines for Birth to 5 Years: Results from the stakeholder consultation. J Phys Act Health 2020;17(1):126-137. https://doi.org/10.1123/jpah.2019-0188

4. Woza, Mtwana. https://www.youtube.com/watch?v=E11vCWPhxNk (accessed 17 June 2021).

5. Draper CE, Silubonde TM, Mukoma G, van Sluijs EMF. Evaluation of the dissemination of the South African 24-hour movement guidelines for birth to 5 years. Int J Environ Res Public Health 2021;18(6):3071. https://doi. org/10.3390/ijerph18063071

6. Tomaz SA, Hinkley T, Jones RA, et al. Screen time and sleep of rural and urban South African preschool children. Int J Environ Res Public Health 2020;17(15):5449. https://doi.org/10.3390/ijerph17155449

7. Rae DE, Tomaz SA, Jones RA, et al. Sleep and BMI in South African urban and rural, high and low-income preschool children. BMC Public Health 2021;21:571. https://doi.org/10.1186/s12889-021-10591-5

S Afr J Child Health 2021;15(2):58-59. https://doi.org/10.7196/SAJCH.2021.v15.i2.1909 